\title{
Purple Sweet Potato Takes on Colon Cancer
}

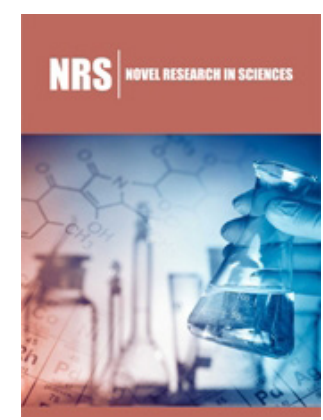

*Corresponding author: Khalid Asadi, Auckland Cancer Society Research Centre, Faculty of Medical and Health Sciences, The University of Auckland, Auckland, New Zealand

Submission: 海July 10, 2019

Published: 海 August 01, 2019

Volume 1 - Issue 4

How to cite this article: Khalid A, Lynnette R F, Martin P, Nishi K. Purple Sweet Potato Takes on Colon Cancer. Nov Res Sci.1(4). NRS.000517.2019.

DOI: 10.31031/NRS.2019.1.000517

Copyright@ Khalid Asadi, This article is distributed under the terms of the Creative Commons Attribution 4.0 International License, which permits unrestricted use and redistribution provided that the original author and source are credited.

\author{
Khalid Asadi ${ }^{1}$, Lynnette R Ferguson ${ }^{1,2}$, Martin Philpott ${ }^{1 *}$ and Nishi Karunasin- \\ ghe $^{1}$
}

${ }^{1}$ Auckland Cancer Society Research Centre, Faculty of Medical and Health Sciences, The University of Auckland, Auckland, New Zealand

${ }^{2}$ Discipline of Nutrition and Dietetics, Faculty of Medical and Health Sciences, The University of Auckland, Auckland, New Zealand

\section{Case Report}

After cardiovascular disease, cancer is the second leading cause of death in the Western world. The World Health Organisation estimates that one third of all occurrences of cancer are preventable, largely through the cessation of smoking, but also by dietary modification and prevention the exposure to carcinogens. (http://www.who.int/cancer/en/). The role of diet in the aetiology of cancer has been established in many epidemiological studies including the protective effect of diets high in fruit and vegetables. Anthocyanin rich foods have been shown to be chemopreventative in many animal models of cancer, and epidemiological evidence suggests they can also function in this role in humans. In my study, the potential chemopreventative properties of Purple Sweet Potato (PSP), called Kumara in New Zealand, which contains high levels of anthocyanins and can realistically be consumed in the human diet in larger quantities than most other anthocyanic foods, were investigated. The ability of PSP to prevent adenoma formation in the APCMIN mouse, which is a genetic model of Colorectal cancer (CRC), along with the potentially underlying mechanisms of action, was examined.

Reductions in polyp numbers in $\mathrm{APC}^{\mathrm{MIN}}$ mice have been reported previously with a variety of pharmaceutical or dietary regimes. Hydroxymatairesinol (HM-3000) was reported to reduce polyp formation in $\mathrm{APC}^{\mathrm{MIN}}$ mice due to the antioxidant activity of HM-3000. Seleniumenriched broccoli reduced the polyp number in $\mathrm{APC}^{\mathrm{MIN}}$ mice by $30.7 \%$, an effect attributed

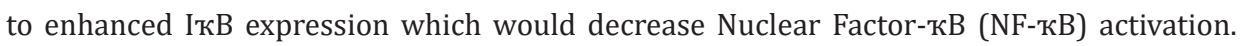
Specific inhibition of Cyclooxginease-2 (COX2) by rofecoxib and inducible Nitric Oxide (iNOS) by aminoguanidine, as well as knockout of these, has been shown to inhibit polyp formation in APC mutant mice. Xenobiotics Metabolized Enzymes (XME) activity has been demonstrated to be modulated by a variety of compounds, including curcumin, which has also been shown to reduce polyposis in $\mathrm{APC}^{\mathrm{MIN}}$ mice.

In the present study, diets supplemented with PSP: flesh, skin or Anthocyanin Rich Extract (ARE) all gave substantial reductions in polyp numbers in $\mathrm{APC}^{\mathrm{MIN}}$ mice of approximately two thirds or more and reduced DNA damage in leukocytes, inhibited NF- $\mathrm{B} \mathrm{activation} \mathrm{in}$ splenocytes, reduced COX2 and iNOS expression in liver tissue, lowered hepatic Cytochrome 450 (CYP450) levels and improved hepatic Glathione S transferase (GST) levels. The individual contributions of all these effects towards the reduction in polyp formation in the APCMIN mouse consuming supplemented diets were observed, and an argument could be made for any one of these effects being responsible for the reduction in polyposis. However, it seems likely that the powerful chemopreventative properties of the PSP supplemented diets in the $\mathrm{APC}^{\mathrm{MIN}}$ mouse are a combination of all these effects, perhaps explaining why the magnitude of polyp reduction is higher than those reported for many other agents. The active compound or compounds in the present Kumara may be modulating each of these effects independently. However, central to the development of polyps in the $\mathrm{APC}^{\mathrm{MIN}}$ mouse is the mutation of the APC gene. APC normally targets $\kappa$-catenin for phosphorylation, ubiquination and degradation, preventing free $к$-catenin from accumulating. If APC is not functional, free К-catenin acts as a transcription factor, initiating expression of a variety of genes. $\kappa$-catenin 
target genes include cyclin-D1, COX2, iNOS and CYP450s. Therefore, APC mutation would promote proliferation by increasing cyclin-D1 levels (cyclin-D1) is a regulator of the cell cycle, and promote inflammation through the induction of COX2 and iNOS expression, which in turn would activate NF- $\mathrm{BB}$. CYP450 expression would be increased, and elevated, CYP450s can drive Radical Oxygen Species (ROS) production.

Thus, restoration of the APC pathway by ARE or supplemented diets, perhaps through inhibition of $к$-catenin, would explain the suppressed growth of colon cells, which have mutant APC via reduced cyclin-D1 levels, the lowered CYP1A activity in mouse livers and, consequently, the antioxidant activity through reduced CYP450-produced ROS. It would also provide a mechanism for the suppression of COX2 and iNOS expression by ARE or supplemented diets, in animal models, which would ameliorate inflammation, in turn reducing NF-кB activation (Figure 1-3). However, the order of events between COX2 and iNOS expression and NF- $\mathrm{KB}$ activation is unclear, since NF- $\mathrm{KB}$ activation is also capable of inducing expression of these genes. The only effect of ARE or supplemented diets that cannot currently be explained by $\mathrm{K}$-catenin inhibition is the increase in GST activity observed in wild-type mice, as no direct link between $\mathrm{K}$-catenin and GST expression could be found in the literature. However, GST is capable of processing COX2 metabolites and various reports have demonstrated an inverse correlation between COX2 and GST expression although the mechanism remains unclear. Hence, PSP modulation of GST may be related to COX2 inhibition.

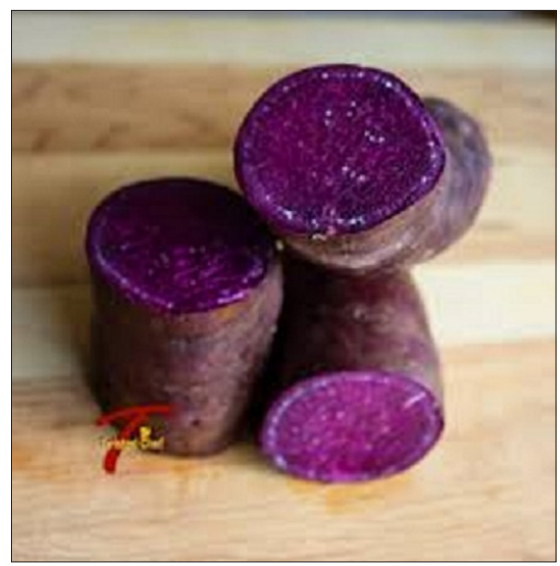

Figure 1

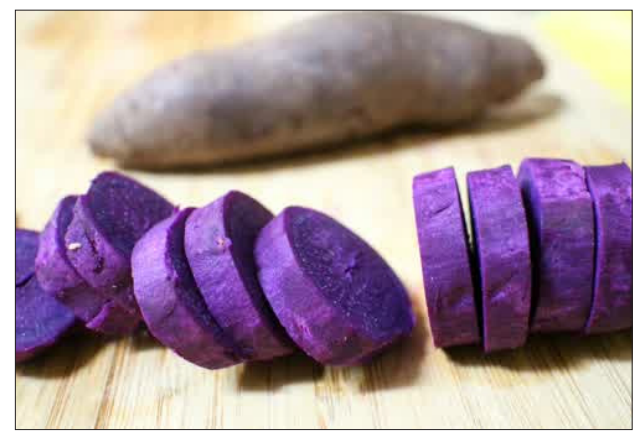

Figure 2

Figure 3

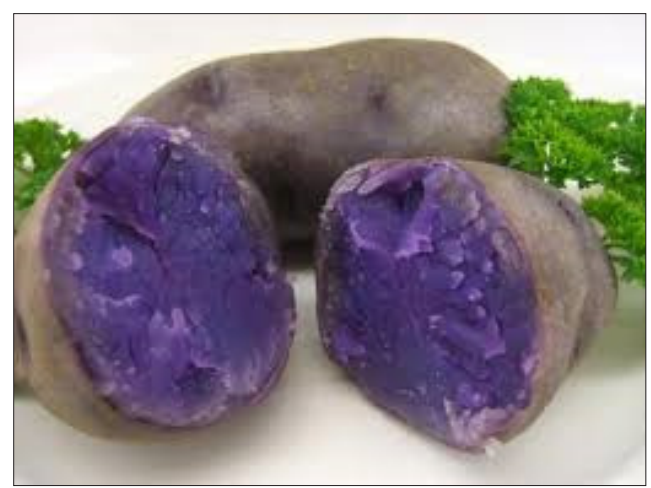


Interestingly, of the flesh-, skin-, or ARE- supplemented diets, the ARE-supplemented diet gave the greatest reduction in polyp numbers (74.9\% v's $66.9 \%$ [flesh] and $65.1 \%$ [skin]), provided the greatest protection against both endogenous and exogenous DNA damage and had the largest modulatory effect on XME. These results show that the chemopreventative activity of resides within the ARE and that this activity is not lost by removal from the biological matrix. In fact, the superior activity of the ARE-supplemented diet suggests bioavailability is increased by the extraction process.

Although strong chemopreventative properties have been demonstrated here for PSP, the distinguishing feature of which is the high anthocyanin pigmentation, the causative agent has not been conclusively identified. Activity was not lost in the ARE-supplemented diet, which otherwise would have eliminated anthocyanins as the active compound. In fact, the ARE-supplemented diet, where anthocyanins have been enriched relative to PSP constituents, had slightly greater activity than the diets supplemented with PSP flesh or skin, which is consistent with anthocyanins being the active compound. Furthermore, although not as effective as PSP, which has higher anthocyanin content, other anthocyanin-rich foods and extracts have been shown to reduce polyp numbers in $\mathrm{APC}^{\mathrm{MIN}}$ mice, and pure anthocyanins have been shown to be strong antioxidants and inhibitors of NF-кB activation, COX2 expression and iNOS expression (Figure $4 \& 5$ ). This, the combined evidence argues strongly that the active compounds in PSP are anthocyanins. However, the possibility that other phenolic compounds that are co-purifying during the extraction process are contributing to, or are solely responsible for, the chemopreventative effects of PSP cannot be completely eliminated.

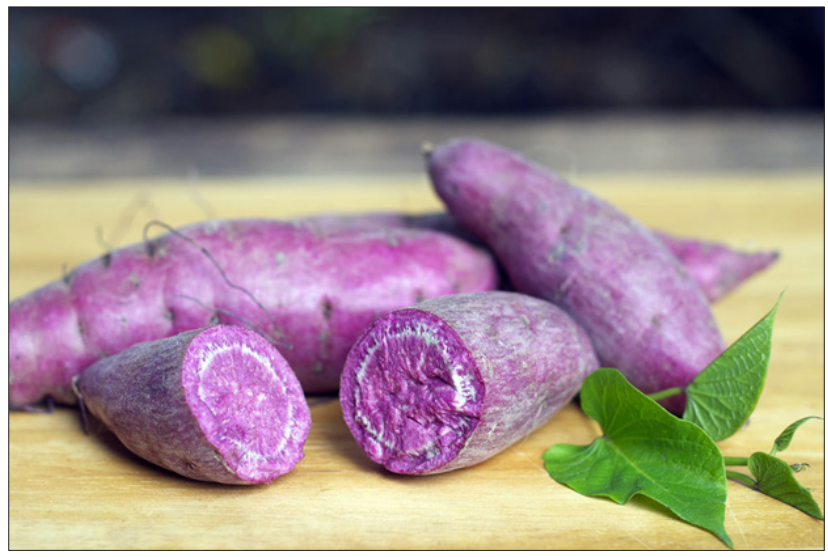

Figure 4

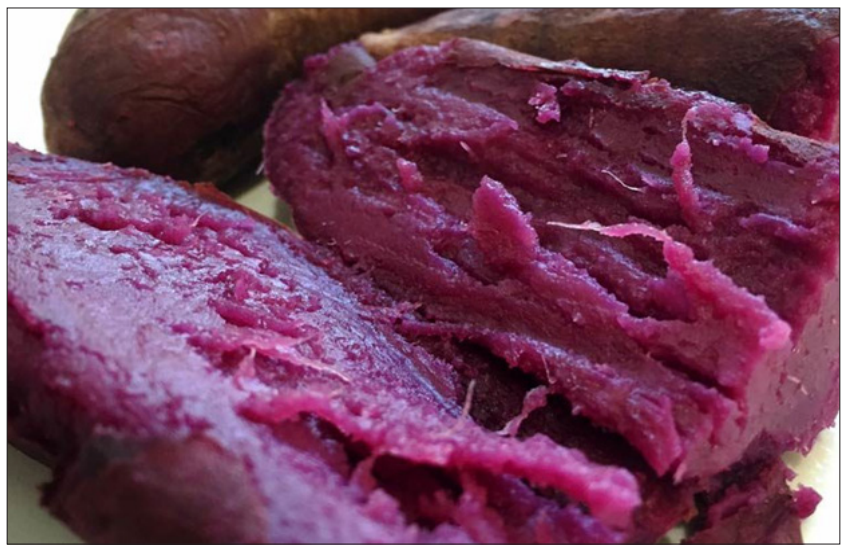

Figure 5

For possible submissions Click below: 